\title{
Oro Cutaneous Fistula of a Periodontal Origin
}

Rajeev Pandey ${ }^{1}$, Rajat Gupta², Nitin Bhagat ${ }^{3}$, J ahnur Ikamul I slam

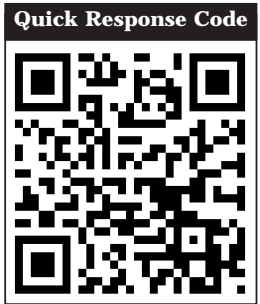

doi: $10.5866 / 2017.9 .10069$

1,2,3Senior Lecturer

${ }^{4} \mathrm{PG}$ student

Department of Oral and Maxillofacial Surgery,

School of Dental Sciences,

Sharda University,

Greater Noida, UP, India

\section{Article Info:}

Received: J anuary 11, 2017

Review Completed: February 12, 2017

Accepted: March 13, 2017

Available Online: March, 2017 (www.nacd.in)

(C) NAD, 2017 - All rights reserved

\section{Email for correspondence:}

rajeevpandeyleo@gmail.com

\begin{abstract}
:
Orocutaneous fistula (OCF) of facial region is uncommon. The most common etiology is odontogenic infection involving pulp. In most cases diagnosis is easy; and specific treatment can be started leading to cure. A case of OCF of posterior mandibular region of periodontal origin is reported here in this paper. The diagnosis became difficult as impacted third molar was totally covered with soft tissue. F istula tract was traced till mandibular third molar. Surgical removal of third molar was performed and excision of sinus tract was done. The treatment leads to complete healing of the fistula site. No reoccurrence was seen after 1 month follow up.
\end{abstract}

Key words: Oro cutaneous fistula, infection, periodontal pocket

\section{INTRODUCTION}

Fistula is defined as an abnormal pathological pathway between two anatomic spaces. It is called as oro cutaneous fistula (OCF) if there is a pathological communication between oral cavity and facial skin.Occurrence of OCF is very rare in this era of antibiotics. If it occurs, the diagnosis presents a challenge. It is misdiagnosed with skin lesions, sweat gland lesions, neoplasms, osteomyelitis, actinomycosis, tuberculosis. ${ }^{1}$ Due to lack of knowl edgethat such a condition can be dueto dental origin few patients may seek treatment from medical fraternity. ${ }^{2}$ The condition becomes more unique if a specific odontogenic cause cannot be determined.Misdiagnosis of such a condition may lead to multiple superficial dermatological surgical operations, unnecessary long term antibiotic therapy. Later the infection become chronic and spread to larger area creating cosmetic deformities. Main etiology for odontogenic oro cutaneous is pulp necrosis due to caries or trauma. ${ }^{3}$ OCF due to periodontal origin is rare. We report a case of OCF of lower right posterior region due to completely soft tissue impacted third molar which was periodontally involved along with first and second molars. 


\section{Case Report:}

A 25 year old male reported with chief complaint of pus discharge and mild pain from right lower posterior mandibular region extraorally since 4 months (Figure 1). Past medical history was not contributory. This was patient first dental visit. The patient had no history of previous trauma to that site. Previously the patient had visited local medical practitioner for the same chief complaint. The patient was told that it was a local skin disease and was prescribed antibiotics and painkillers. This provided intermittent relief for few days. Dental history also revealed that patient had swelling and trismus in the right mandibular posterior region before development of oro cutaneous fistula. On clinical examination: An extraoral pus draining opening was present measuring $1 \mathrm{~cm} \times 1 \mathrm{~cm}$ about 3 $\mathrm{cm}$ above right angle region. Gentle pressure on the surrounding tissueelicited thick purulent drainage. Dimpling of surrounding skin was seen with reddish discoloration around the opening. There was no regional lymphoadenopathy.

On intraoral examination, mouth opening was mildly restricted with poor oral hygiene and moderate amount of stain and calculus was present. Root stump was present in relation to 37 and grossly decayed 18. On periodontal examination there were deep pockets in the posterior region of upper and lower jaws. There was moderate calculus and deep pockets in the fourth quadrant in relation to 46, 47. Tooth 48 was clinically absent. $X$ ray orthopantogram was advised along with complete blood count, bleeding time, clotting time and HIV status.All blood parameters were within normal range and HIV status was negative.

$X$ ray findings: vertically impacted 48 with radiolucency at apex of mesial root and distal to crown (F igure2). As no carious teeth were detected, we concluded that oro cutaneous fistula was of periodontal origin due to pockets in 46 and 47 region involving 48.

\section{Treatment:}

Surgical removal of 48 and complete excision of the oro cutaneous fistula was planned. Before surgical treatment was initiated patient was started on oral antibiotics and scaling was done. After standard intra oral and extra oral skin and mucosa preparation, sterile draping was done.The surgical removal of 48 was done using modified Wards incision. The mucoperiosteal flap was elevated which had become fibrotic due to the chronic infection. 48 was exposed. A thick follicular tissue was present distal to 48. 48 and follicular tissue was removed without need for removal of any bone (Figure 3). Toilet of the socket was done using povidone iodine and bone margins were smoothen using bone file. After giving local infiltration around the fistula, the tract was traced using straight periosteal elevator till the buccal vestibule of impacted 48 region.For removal of fistula tract an elliptical incision was placed. Sharp incision was placed through skin and sub cutaneous tissue. After that blunt dissection was done following around the tract through the masseter muscle. The whole tract till the intraoral mucosa was removed (Figure 4). Closure was done using 3-0 vicryl and 3-0 mersilk sutures. Suture removal was done after 1 week without any recurrence and discharge (Figure 5).

\section{DISCUSSION:}

In the case presented the clinical diagnosis was a challenge. Diagnosis of OCF was difficult in absence of carious tooth and history was negative for any trauma to region involved. The etiology of oro cutaneous fistula are many, therefore a multiple specialist hel $p$ has to be seeken to arrive at definitive diagnosis. If such a lesion is not treated early it can spread causing cosmetic defects of the facial skin and may spread to facial spaces and can cause osteomyelitis of the bone involved.Less than 50\% patients have history of tooth pain. Mandible is more commonly involved and the most common sites are chin and angle of jaw. The presentation of the skin opening of OCF can be nodular, scar like, ulcer or abscess. In the above case the presentation was nodular. OCF due to periodontal cause is rare. The pathogenesis of the above case may be due to poor oral hygiene had lead to deep periodontal pocket leading to infection foci accumulating in the mucoperiodontal flap in respect to $46,47,48$ region. As 48 was totally soft tissue impacted this had lead to spread of infection to periapical region of 48 and spreading laterally involving buccal space. As the patient did not took any treatment during acute phase the infection spread more laterally leading to perforation of skin and formation of sinus which epithelialised leading to fistula formation. As the patient had no dental pain he was unaware that the origin of infection was odontogenic and he consulted medical practioner. Alsolack of knowledge of medical fraternity about odontogenic infection as 


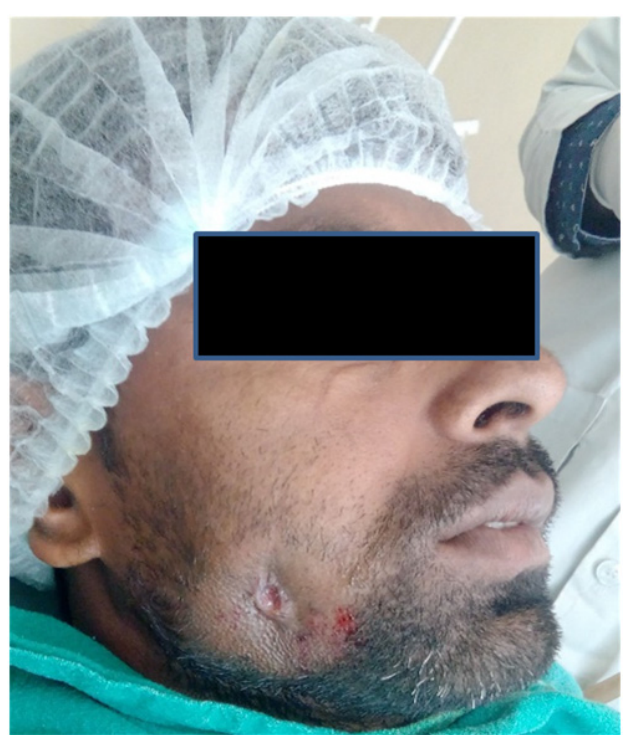

Figure 1: Clinical picture showing pus discharge from right lower posterior mandibular region.

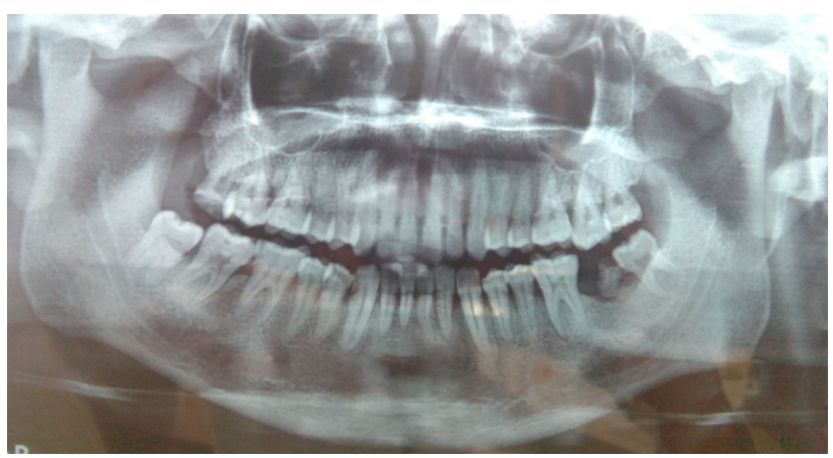

Figure 2: Orthopantogram (OPG) showing vertically impacted 48 with radiolucency at apex of mesial root and distal to crown.

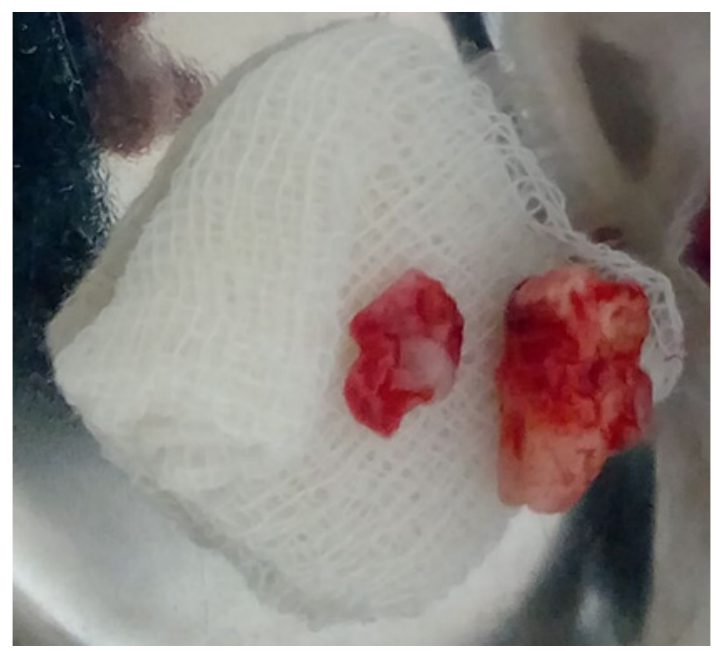

Figure 3: Excision of 48 and follicular tissue without removal of any bone

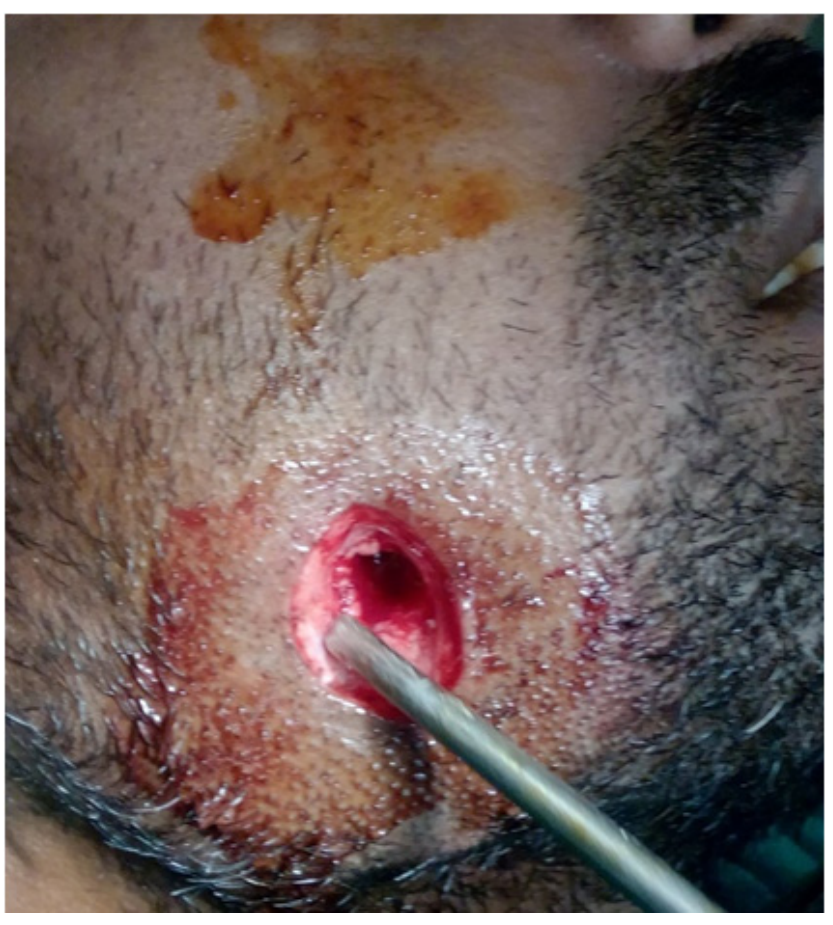

Figure 4: Removal of the whole tract till the intraoral mucosa.

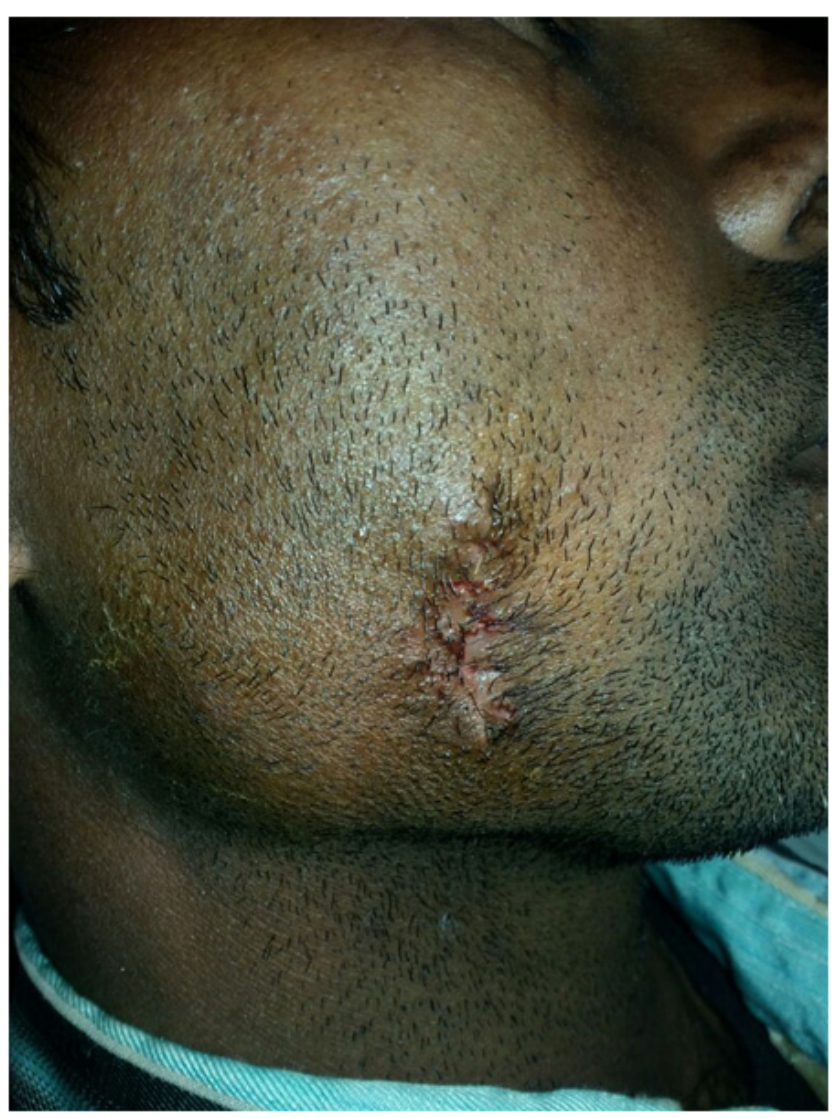

Figure 5: Clinical picture showing no recurrence and no discharge. 
etiology of OCF had lead to chronicity of the infection. The most reliable factor to determine the offending tooth is to follow the fistural tract through extraoral opening and seen where the tract ends. In the above case report also the offending tooth was diagnosed by this method. Various authors have reported that the removal or endodontic treatment of offending tooth can lead to healing of the fistula without removal of the fistula. ${ }^{4}$ B ut the time period taken for this is very long also the cosmetic defect of the skin is not corrected. In the present case the removal of impacted 48 was donealong with excision of the fistula to fastened the healing and correct the skin deformity and prevent the reoccurrence. Past history plays a very important role in differentiating lesions from odontogenic origin or any other etiology. In the present case also patient had history of space infection due to odontogenic origin with trismus. Location of fistula in most of times is adjacent to the causativetooth. This was al so seen in the present case. Diagnosis is confirmatory by clinical diagnosis in most cases if etiology is odontogenic. In the present case clinical diagnosis was not confirmatory because periodontal pocket leading to spread of infection causing OCF is very rare, so additional radiographic diagnostic aids were used. Other diagnostic aids are: CBCT (Cone Beam Computed Tomogram), Pulp vitality test, CT fistulography. ${ }^{5}$ In review of literature, the time taken to confirm diagnosis may delayed in months sometimes even longer. ${ }^{6}$

\section{CONCLUSION}

Diagnosis of OCF is a challenge for both dental and medical fraternity. The case report highlights the importance of early dental examination in all cases of OCF to prevent misdiagnosis. Also for the dental practitioner it highlights the importance of periodontal examination to rule out as etiological factor if a definitive odontogenic etiology cannot be determined.

\section{REFERENCES}

1. Tulingulec A, Sec,kin D, Bulut S, Sarfakoglu E. Cutaneous sinus tract of dental origin. Int J Dermatol 2001; 40:650 -2.

2. Vermani M, Kalia V, Singh S, Garg S, Aggarwal S, Khurana R, Kalra G. Orocutaneous Fistula or Traumatic Infectious Skin Lesion: A Diagnostic Dilemma. Case Rep Dent 2015; 353.

3. Samir N, Al-Mahrezi A, Al-Sudairy S. Odontogenic cutaneous fistula: report of two cases. Sultan Qaboos Univ Med J 2011; 11:115-8.

4. Abdelmoumen E, Skhiri SZ, Boughzela A. Non surgical management of cutaneous sinus tract of odontogenic origin: A case report. J Dent Oral Hyg 2014; 6:30-5.

5. Ranga U, Aiyappan SK, Veeraiyan S. A Case of Intermittently Discharging Skin Lesion: Orodentocutaneous Fistula Demonstrated on CT Fistulography. J Clin Diagn Res 2014; 8:09-11

6. Guevara-Gutiérrez E, Riera-Leal L, Gómez-Martínez M, Amezcua-Rosas G, Chávez-Vaca CL, Tlacuilo-Parra A. Odontogenic cutaneous fistulas: clinical and epidemiologic characteristics of 75 cases. Int J Dermatol 2015; 54:50-5

\section{Gain quick access to our journal online View our journal at www.nacd.in}

